\title{
Second Derivative Approximation for Origin-Based Algorithm
}

\author{
Feng Li \\ IBM China Research Laboratory, \\ Beijing 100193, P.R.China \\ lfeng@cn.ibm.com
}

\begin{abstract}
Origin-based algorithm(OBA) for traffic assignment problem has been demonstrated preferable to the widely accepted and used Frank-Wolfe algorithm and path-based algorithms. Note that OBA can not avoid path enumeration of concerned network, which will lead to two disadvantages. One is the intensive memory requirements and the other is the difficulties in manipulating and storing paths. In order to solve these problems, we first propose the lower and upper bounds of the Hessian matrix, which can be calculated without path enumeration. Then use the lower bound of Hessian matrix to approximate the direction of the origin-based algorithm. According to our computational results, the modified origin-based algorithm(MOBA) improves the convergence performance greatly. The results indicate that MOBA can deliver better and more reliable convergence than OBA and saves much more CPU time especially when large-scale networks are being considered.
\end{abstract}

Keywords: Traffic Assignment, Origin-based Algorithm, Second Derivative, User Equilibrium.

\section{Introduction}

Traffic assignment problem (TAP) is the key problem for the long term planning and evaluation of urban transportation network. It is to assign the traffic flows of each OD (Origin-Destination) pair to links of urban transportation network in terms of certain principle, and count out all link traffic flows. There are many principles for TAP, but the most often used principles are the first and second principles of Wardrop, i.e. User Equilibrium principle and System Optimum principle 11. In order to find a solution satisfying the User Equilibrium principle, Beckmann et al. proposed a convex mathematical programming [2] [3] which became the main tool for solving traffic assignment problem. Since the work of Beckmann et al, many algorithms have been suggested to solve it. All of them can be broadly divided into three categories according to the solution space the algorithm resides, i.e., the link- path- or origin-based algorithms.

The link-based algorithms, including Frank-Wolfe (FW) algorithm given by LeBlanc et al. 4] and several modified FW algorithms [5] 6] 7] 8] 9, yield linkbased solutions. The path-based algorithms, including disaggregated simplicial 
decomposition (DSD) algorithm [10], gradient projection (GP) algorithm [11] [12] and conjugate gradient projection (CGP) algorithm [13] etc., can determine both an aggregate link-based solution and an individual path-based solution which is not available for link-based algorithms. The origin-based algorithm(OBA) 14 can provide both an aggregate link-based solution and a constructive path-based solution. In the past, path-based algorithms and origin-based algorithm were not considered as a viable solution approach for large-scale network problems because of intensive memory requirements and difficulties in manipulating and storing paths. However, path-based algorithms and origin-based algorithm are now made possible due to the dramatic advances in computing technology.

Recent researches [13] 14 have shown and demonstrated that the origin-based algorithm excels not only the FW algorithm but also some path-based algorithms such as DSD, GP and CGP. Although OBA was designed for solutions with high levels of accuracy, it also can not avoid manipulating and storing paths in each iteration of the algorithm. Plentiful computational experiments of OBA also indicate that most of the $\mathrm{CPU}$ time of the OBA is to enumerate and manipulate paths of the restricting subnetwork $A_{p}(\alpha)$ (see [14]).

Motivated by the problems discussed above, we give a second derivative approximation to modify the origin-based algorithm. By using this approximation, the modified origin-based algorithm (MOBA) can avoid enumerating and manipulating paths of the restricting subnetworks and outperforms OBA in the speed of convergence and memory requirements. The remaining sections of this paper are organized as follows. Section 2 describes the traffic assignment problem and introduces the origin-based algorithm. The modified origin-based algorithm by using second derivative approximation is then proposed in section 3. Section 4 describes the computational results generated by OBA and MOBA from two real networks including Sioux Falls and Barcelona network. Finally, a brief conclusion is given in section 5 .

\section{User-Equilibrium Traffic Assignment and Origin-Based Algorithm}

Before introducing the user equilibrium traffic assignment model and the originbased algorithm, we give the following notations which are used in this paper.

$\begin{array}{ll}G & G=(\mathcal{N}, \mathcal{A}) \text { represent an urban transportation network } \\ \mathcal{N} & \mathcal{N}=(1,2, \cdots, N) \text { the set of nodes } \\ \mathcal{A} & \text { the set of all directed links } \\ O & \text { the set of origin nodes } \\ D & \text { the set of destination nodes } \\ a & a=\left[a_{t}, a_{h}\right] \text { the directed link of the network } \\ a_{t} & \text { the tail node of link } a \\ a_{h} & \text { the head node of link } a \\ x_{a} & \text { the traffic flow on link } a \\ c_{a} & \text { the traffic cost on link } a\end{array}$




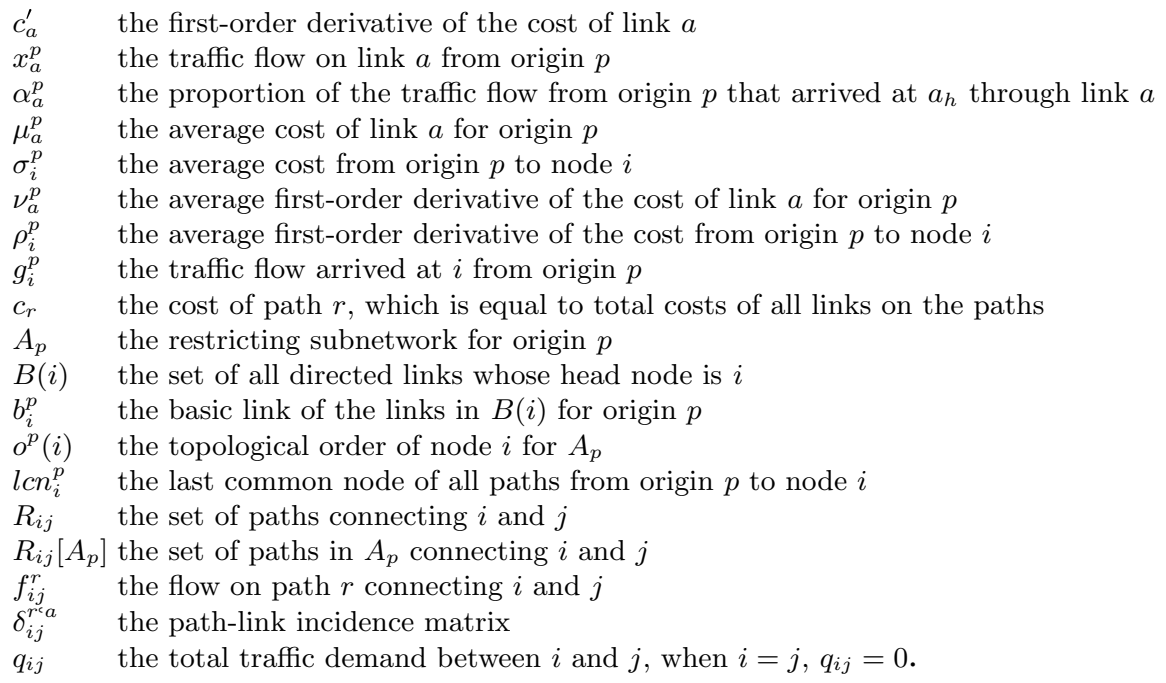

\subsection{User Equilibrium Formulation}

It is well known that TAP can be formulated as an optimization program with a nonlinear objective function and linear constraints. Consider an urban transportation network represented by a directed graph $G=(\mathcal{N}, \mathcal{A})$. For convenience, we suppose $O=D=\mathcal{N}$. Using the above notes, the User Equilibrium (UE) traffic assignment problem can be written as

$$
\begin{array}{lll}
\min & Z=\sum_{a \in \mathcal{A}} \int_{0}^{x_{a}(\alpha)} c_{a}(\varpi) d \varpi & \\
\text { s.t. } & \sum_{\substack{a \in B(i) \\
\alpha_{a}^{p} \geq 0,}} \alpha_{a}^{p}=1, & 1 \leq i, p \leq N, i \neq p \\
& \forall a \in \mathcal{A}, 1 \leq p \leq N
\end{array}
$$

where $\alpha=\left(\alpha^{1}, \alpha^{2}, \cdots, \alpha^{N}\right), \alpha^{p}=\left(\cdots, \alpha_{a}^{p}, \cdots\right), a \in \mathcal{A}$ is the variable of the mathematical programming, and $\alpha_{a}^{p}$ is defined as,

$$
\alpha_{a}^{p}=\frac{x_{a}^{p}}{\sum_{b \in B\left(a_{h}\right)} x_{b}^{p}}
$$

especially when the total traffic flow from origin $p$ that arrived at $a_{h}$ is zero, we let any one of the proportions of those links with head node $a_{h}$ be one and the others be zero. $x_{a}(\alpha)$ is defined as,

$$
x_{a}(\alpha)=\sum_{i=1}^{N} x_{a}^{i}(\alpha)=\sum_{i=1}^{N} \sum_{j=1}^{N} \sum_{r \in R_{i j}} f_{i j}^{r} \cdot \delta_{i j}^{r, a}=\sum_{i=1}^{N} \sum_{j=1}^{N} \sum_{r \in R_{i j}} q_{i j} \prod_{b \subseteq r} \alpha_{b}^{i} \delta_{i j}^{r, a}
$$

For each origin $p$ and every node $j \neq p$ we choose one link $b_{j}^{p} \in A_{p}:\left(b_{j}^{p}\right)_{h}=j$ as the basic link of the links with head node $j$ for origin $p$, call all other links 
with head node $j$ in the restricting subnetwork $A_{p}$ (if there are any) the nonbasic links for origin $p$ and denote them as $\operatorname{NB}_{j}^{p}=\left\{a \in A_{p}: a_{h}=j ; a \neq b_{j}^{p}\right\}$; $\mathrm{NB}^{p}=\cup_{j \in \mathcal{N} ; j \neq p} \mathrm{NB}_{j}^{p}$. Then $\alpha$ can be viewed as a function of $\alpha^{\mathrm{NB}}$ :

$$
\begin{array}{ll}
\alpha_{b_{j}^{p}}^{p}\left(\alpha^{\mathrm{NB}}\right)=1-\sum_{a \in \mathrm{NB}_{j}^{p}} \alpha_{a}^{p \mathrm{NB}}, & \forall j \in \mathcal{N} \backslash\{p\}, \forall p \in \mathcal{N} \\
\alpha_{a}^{p}\left(\alpha^{\mathrm{NB}}\right)=\alpha_{a}^{p \mathrm{NB}}, & \forall a \in \mathrm{NB}^{p}, \forall p \in \mathcal{N} \\
\alpha_{a}^{p}\left(\alpha^{\mathrm{NB}}\right)=\alpha_{a}^{p \mathrm{NB}}=0, & \forall a \in \mathcal{A} \backslash A_{p}, \forall p \in \mathcal{N}
\end{array}
$$

Using (4), (11) can be simply changed into,

$$
\begin{array}{ll}
\min & Z=\sum_{a \in \mathcal{A}} \int_{0}^{x_{a}\left(\alpha^{\mathrm{NB}}\right)} c_{a}(\varpi) d \varpi \\
\text { s.t. } & \alpha_{a}^{p \mathrm{NB}} \geq 0, \quad \forall a \in \mathcal{A}, 1 \leq p \leq N
\end{array}
$$

It is simply to prove that (1) equals to the famous Beckmann's transformation [2. Convergence algorithms for solving it have been studied since the 1960s.

\subsection{Origin-Based Algorithm}

The origin-based algorithm operates directly on the space of the traffic flow proportion. It makes successive moves as an approximate Newton direction at each iteration. The main body of the algorithm can be found in [14.

\section{Modified Origin-Based Algorithm}

Our plentiful computational experiments and investigation of OBA indicate that there is no efficient and quick methods to find the last common nodes of the restricting subnetwork $A_{p}(\alpha)$ and we cannot but enumerate all paths of the restricting subnetwork in the algorithm. We also found the last common nodes were only used to calculate the approximation of the Hessian matrix. Hence if we can propose a new Hessian matrix approximation without using the last common nodes to replace that of the OBA, we will avoid path enumeration. Below the lower bound and upper bound of the Hessian matrix will be calculated firstly, then a novel Hessian matrix approximation is determined by these bounds. The novel approximation will be used to modify the origin-based algorithm.

Before giving the bounds of the Hessian matrix, we need the following definitions and propositions.

Definition 1. For any origin $p$, and any two nodes $i$ and $j$, we define the following formal expression as the proportion of the flow from origin $p$ that arrives at node $j$ through node $i$.

$$
\chi_{i \rightarrow j}^{p}= \begin{cases}\sum_{r \in R_{i j}\left[A_{p}\right]}\left(\prod_{a \subseteq r} \alpha_{a}^{p}\right), & \forall p \in \mathcal{N}, i \neq j \\ 1, & i=j\end{cases}
$$


Definition 2. For any origin $p$ and node $j$, the following equation is defined as the average cost from origin $p$ to node $j$.

$$
\sigma_{j}^{p}=\sum_{r \in R_{p j}\left[A_{p}\right]: a \subseteq r} c_{r} \cdot \prod_{a \subseteq r} \alpha_{a}^{p}
$$

Definition 3. For any origin $p$ and link $a$, the following equation is defined as and the average cost of link a for origin $p$.

$$
\mu_{a}^{p}=\sum_{r \in R_{p a_{h}}\left[A_{p}\right]: a \subseteq r} c_{r} \cdot \prod_{a^{\prime} \subseteq r: a^{\prime} \neq a} \alpha_{a^{\prime}}^{p}=c_{a}+\sigma_{a_{t}}^{p}
$$

Definition 4. For any origin $p$, the topological order of all nodes in $A_{p}$ is defined as a one-to-one function $o: \mathcal{N} \rightarrow\{1,2, \cdots, N\}$ such that, $\forall a=[i, j] \in A_{p} \Rightarrow$ $o^{p}(i)<o^{p}(j)$, and particularly $o^{p}(p)=1$.

Definition 5. The total flow arrived at node $j$ from origin $p$ is defined as,

$$
g_{j}^{p}=\sum_{i=1}^{N} \sum_{\substack{r \in R_{p i}\left[A_{p}\right] ; \\ j \in r}} q_{p i} \prod_{a \subseteq r} \alpha_{a}^{p}=\sum_{i=1}^{N} q_{p i} \cdot \chi_{p \rightarrow j}^{p} \cdot \chi_{j \rightarrow i}^{p}=\sum_{i=1}^{N} q_{p i} \cdot \chi_{j \rightarrow i}^{p}, \quad \forall p \in \mathcal{N}
$$

Using these definitions, the following equations can be easily gotten,

$$
\begin{aligned}
& \sigma_{j}^{p}=\sum_{a \in A_{p}: a_{h}=j} \alpha_{a}^{p} \cdot \sum_{r \in R_{p j}\left[A_{p}\right]: a \subseteq r} c_{r} \cdot \prod_{a^{\prime} \subseteq r: a^{\prime} \neq a} \alpha_{a^{\prime}}^{p}=\sum_{a \in A_{p}: a_{h}=j} \alpha_{a}^{p} \cdot \mu_{a}^{p} \\
& \mu_{a}^{p}=c_{a}+\sum_{r \in R_{p a_{t}}\left[A_{p}\right]: a \subseteq r} c_{r} \cdot \prod_{a^{\prime} \subseteq r} \alpha_{a^{\prime}}^{p} \\
& =c_{a}+\sum_{r \in R_{p a t}\left[A_{p}\right]: a \subseteq r} \prod_{a a^{\prime} \subseteq r} \alpha_{a^{\prime}}^{p} \cdot \sum_{e \subseteq r} c_{e} \\
& =c_{a}+\sum_{a^{\prime} \in A_{p}} c_{a^{\prime}} \cdot \chi_{p \rightarrow a_{t}^{\prime}}^{p} \cdot \alpha_{a^{\prime}}^{p} \cdot \chi_{a_{h}^{\prime} \rightarrow a_{t}}^{p} \\
& =c_{a}+\sum_{a^{\prime} \in A_{p} ; o^{p}\left(a_{h}^{\prime}\right)<o^{p}\left(a_{h}\right)} c_{a^{\prime}} \cdot \alpha_{a^{\prime}}^{p} \cdot \chi_{a_{h}^{\prime} \rightarrow a_{t}}^{p} \\
& \frac{\partial x_{a^{\prime}}}{\partial \alpha_{a}^{p}}=\sum_{i=1}^{N} \sum_{r \in R_{p i}\left[A_{p}\right] ; a^{\prime} \subseteq r ; a \subseteq r} q_{p i} \cdot \prod_{e \subseteq r ; e \neq a} \alpha_{e}^{p} \\
& = \begin{cases}\sum_{i=1}^{N} q_{p i} \sum_{r \in R_{p i}\left[A_{p}\right] ; a \subseteq r ; a^{\prime}=a} \prod_{e \subseteq r ; e \neq a} \alpha_{e}^{p}=g_{a_{h}}^{p}, & a^{\prime}=a \\
0, & a_{h}^{\prime}=a_{h}, a^{\prime} \neq a \\
\alpha_{a^{\prime}}^{p} \cdot \chi_{a_{h}^{\prime} \rightarrow a_{t}}^{p} \cdot g_{a_{h}}^{p}, & o^{p}\left(a_{h}^{\prime}\right)<o^{p}\left(a_{h}\right) \\
\chi_{a_{h} \rightarrow a_{t}^{\prime}}^{p} \cdot \alpha_{a^{\prime}}^{p} \cdot g_{a_{h}^{\prime}}^{p}, & o^{p}\left(a_{h}^{\prime}\right)>o^{p}\left(a_{h}\right)\end{cases}
\end{aligned}
$$




$$
\frac{\partial x_{a^{\prime}}}{\partial \alpha_{a}^{p \mathrm{NB}}}= \begin{cases}g_{a_{h}}^{p}, & a^{\prime}=a \\ -g_{a_{h}}^{p} & a^{\prime}=b_{a_{h}}^{p} \\ 0, & a_{h}^{\prime}=a_{h}, a^{\prime} \neq a, a^{\prime} \neq b_{a_{h}}^{p} \\ \alpha_{a^{\prime}}^{p} \cdot g_{a_{h}}^{p} \cdot\left(\chi_{a_{h}^{\prime} \rightarrow a_{t}}^{p}-\chi_{a_{h}^{\prime} \rightarrow b_{a_{t}}^{p}}^{p}\right), & o^{p}\left(a_{h}^{\prime}\right)<o^{p}\left(a_{h}\right) \\ 0, & o^{p}\left(a_{h}^{\prime}\right)>o^{p}\left(a_{h}\right)\end{cases}
$$

Hence the first-order and second-order derivatives of the variables $\alpha^{\mathrm{NB}}$ can be describe as,

$$
\begin{aligned}
& \frac{\partial Z}{\partial \alpha_{a}^{p \mathrm{NB}}}=\sum_{a^{\prime} \in \mathcal{A}} \frac{\partial Z}{\partial x_{a^{\prime}}} \cdot \frac{\partial x_{a^{\prime}}}{\partial \alpha_{a}^{p \mathrm{NB}}}=\sum_{a^{\prime} \in \mathcal{A}} c_{a^{\prime}} \cdot \frac{\partial x_{a^{\prime}}}{\partial \alpha_{a}^{p \mathrm{NB}}} \\
& =c_{a} \cdot g_{a_{h}}^{p}-c_{b_{a_{h}}^{p}} \cdot g_{a_{h}}^{p}+\sum_{\substack{a^{\prime} \in A_{p} ; \\
o^{p}\left(a_{h}^{\prime}\right)<o^{p}\left(a_{h}\right)}} c_{a^{\prime}} \cdot \alpha_{a^{\prime}}^{p} \cdot g_{a_{h}}^{p} \cdot\left(\chi_{a_{h}^{\prime} \rightarrow a_{t}}^{p}-\chi_{a_{h}^{\prime} \rightarrow b_{a_{h}}^{p}}^{p}\right) \\
& =g_{a_{h}}^{p}\left(\mu_{a}^{p}-\mu_{b_{a_{h}}^{p}}^{p}\right) \\
& \frac{\partial^{2} Z}{\partial \alpha_{a}^{p \mathrm{NB}^{2}}}=\sum_{a^{\prime} \in \mathcal{A}}\left[\frac{\partial^{2} Z}{\partial x_{a^{\prime}}{ }^{2}} \cdot\left(\frac{\partial x_{a^{\prime}}}{\partial \alpha_{a}^{p \mathrm{NB}}}\right)^{2}+\frac{\partial Z}{\partial x_{a^{\prime}}} \cdot\left(\frac{\partial^{2} x_{a^{\prime}}}{\left.\partial \alpha_{a}^{p \mathrm{NB}}\right)}\right]=\sum_{a^{\prime} \in \mathcal{A}} c_{a^{\prime}}^{\prime} \cdot\left(\frac{\partial x_{a^{\prime}}}{\partial \alpha_{a}^{p \mathrm{NB}}}\right)^{2}\right. \\
& =\left(c_{a}^{\prime}+c_{b_{a_{h}}^{\prime}}^{\prime}\right) \cdot g_{a_{h}}^{p}{ }^{2}+\sum_{\substack{a^{\prime} \in A_{p} ; \\
o^{p}\left(a_{h}^{\prime}\right)<o^{p}\left(a_{h}\right)}} c_{a^{\prime}}^{\prime} \cdot \alpha_{a^{\prime}}^{p} \cdot g_{a_{h}}^{p} \cdot\left(\chi_{a_{h}^{\prime} \rightarrow a_{t}}^{p}-\chi_{a_{h}^{\prime} \rightarrow b_{a_{h}}^{p}}^{p}\right)^{2}(15)
\end{aligned}
$$

Notice that,

$$
\chi_{p \rightarrow i}^{p} \cdot \chi_{i \rightarrow j}^{p}=\sum_{r \in R_{p j}\left[A_{p}\right] ;} \prod_{i \in r} \alpha_{a}^{p} \leq \sum_{r \in R_{p j}\left[A_{p}\right]} \prod_{a \subseteq r}=\chi_{p \rightarrow j}^{p}
$$

and for any node $j$ and any origin $p$, it is easy to prove $\chi_{p \rightarrow j}^{p}=1$ by Lemma 4 of [14, then,

$$
0 \leq \chi_{i \rightarrow j}^{p} \leq 1, \quad \forall p \in \mathcal{N}, 1 \leq i, j, \leq N
$$

Hence the upper bound of the Hessian matrix will be,

$$
\frac{\partial^{2} Z}{\partial \alpha_{a}^{p \mathrm{NB} 2}} \leq c_{a}^{\prime} \cdot g_{a_{h}}^{p{ }^{2}}+c_{b_{a_{h}}^{p}}^{\prime} \cdot g_{a_{h}{ }^{2}}^{{ }^{2}}+\sum_{\substack{a^{\prime} \in A_{p} ; \\ o^{p}\left(a_{h}^{\prime}\right)<o^{p}\left(a_{h}\right)}} c_{a^{\prime}}^{\prime} \cdot \alpha_{a^{\prime}}^{p^{2}} \cdot g_{a_{h}}^{p^{2}}
$$

Because,

$$
\begin{aligned}
& \frac{\partial^{2} Z}{\partial \alpha_{a}^{p \mathrm{NB}^{2}}}=c_{a}^{\prime} \cdot g_{a_{h}}^{p^{2}}+c_{b_{a_{h}}^{p}}^{\prime} \cdot g_{a_{h}}^{p^{2}} \\
& +\sum_{a^{\prime} \in A_{p} ; o^{p}\left(a_{h}^{\prime}\right)<o^{p}\left(a_{h}\right)} c_{a^{\prime}}^{\prime} \cdot \alpha_{a^{\prime}}^{p 2} \cdot g_{a_{h}}^{p 2} \cdot\left(\chi_{a_{h}^{\prime} \rightarrow a_{t}}^{p}-\chi_{a_{h}^{\prime} \rightarrow b_{a_{h}}^{p}}^{p}\right)^{2} \\
& \geq c_{a}^{\prime} \cdot g_{a_{h}}^{p^{2}}+\sum_{a^{\prime} \in A_{p} ; a^{\prime} \in B\left(a_{t}\right)} c_{a^{\prime}}^{\prime} \cdot \alpha_{a^{\prime}}^{p^{2}} \cdot g_{a_{h}}^{p^{2}} \cdot\left(1-\chi_{a_{t} \rightarrow b_{a_{h}}^{p}}^{p}\right)^{2} \\
& +c_{b_{a_{h}}^{p}}^{\prime} \cdot g_{a_{h}}^{p^{2}}+\sum_{a^{\prime} \in A_{p} ; a^{\prime} \in B\left(b_{a_{h}}^{p}\right)} c_{a^{\prime}}^{\prime} \cdot \alpha_{a^{\prime}}^{p^{2}} \cdot g_{a_{h}{ }^{2}}^{p^{2}} \cdot\left(\chi_{b_{a_{h}}^{p} \rightarrow a_{t}}^{p}-1\right)^{2}(17)
\end{aligned}
$$


and there is no loop for any restricting network $A_{p}$, hence

$$
\begin{aligned}
& \left.\chi_{a_{t} \rightarrow b_{a_{h} t}^{p}}^{p}=\alpha_{\left[a_{t}, b_{a_{h t}}^{p}\right]}^{p}\right] \\
& \chi_{b_{a_{h t} \rightarrow a_{t}}^{p}}^{p}=\alpha_{\left[b_{a_{h}}^{p}, a_{t}\right]}^{p}
\end{aligned}
$$

Therefore the lower bound of the Hessian matrix is

$$
\begin{aligned}
& \frac{\partial^{2} Z}{\partial \alpha_{a}^{p \mathrm{NB}^{2}}} \geq c_{a}^{\prime} \cdot g_{a_{h}}^{p{ }^{2}}+\sum_{\substack{a^{\prime} \in A_{p} ; \\
a^{\prime} \in B\left(a_{t}\right)}} c_{a^{\prime}}^{\prime} \cdot \alpha_{a^{\prime}}^{p^{2}} \cdot g_{a_{h}}^{p^{2}} \cdot\left(1-\alpha_{\left[a_{t}, b_{a_{h}}^{p}\right]}^{p}\right)^{2} \\
& +c_{b_{a_{h}}^{p}}^{\prime} \cdot g_{a_{h}}^{p{ }^{2}}+\sum_{\substack{a^{\prime} \in A_{p} ; \\
a^{\prime} \in B\left(b_{a_{h}}^{p}\right)}} c_{a^{\prime}}^{\prime} \cdot \alpha_{a^{\prime}}^{p} \cdot g_{a_{h}}^{p} \cdot\left(\alpha_{\left[b_{a_{h}}^{p}, a_{t}\right]}^{p}-1\right)^{2} \\
& \geq c_{a}^{\prime} \cdot g_{a_{h}}^{p{ }^{2}}+c_{b_{a_{h}}^{p}}^{\prime} \cdot g_{a_{h}}^{p^{2}}
\end{aligned}
$$

Using the bounds of the Hessian matrix, we can get the following approximation of Newton direction of the variables $\alpha^{\mathrm{NB}}$,

$$
\Delta d_{a}^{n}=\frac{\mu_{b_{a_{t}}^{n}}^{n}-\mu_{a}^{n}}{\left(c_{a}^{\prime}+c_{b_{a_{t}}^{n}}^{\prime}\right) \cdot g_{a_{t}}^{n}}, \forall a \in \mathrm{NB}^{n}, n \in D .
$$

Thus for any origin $p$, we have,

$$
\begin{array}{ll}
\Delta \alpha_{a}^{p \mathrm{NB}}=\left\{\begin{array}{ll}
\max \left\{-\alpha_{a}^{p \mathrm{NB}}, \lambda \cdot \frac{\mu_{b_{h}^{p}}^{p}-\mu_{a}^{p}}{\left(c_{a}^{\prime}+c_{b_{a_{h}}^{p}}^{\prime}\right) \cdot g_{j}^{p}\left(\alpha^{\mathrm{NB}}\right)}\right\}, & g_{a_{h}}^{p}\left(\alpha^{\mathrm{NB}}\right)>0 \\
-\alpha_{a}^{p \mathrm{NB}}, & g_{a_{h}}^{p}\left(\alpha^{\mathrm{NB}}\right)=0
\end{array}, \quad \forall a \in \mathrm{NB}^{p}\right. \\
\Delta \alpha_{a}^{p \mathrm{NB}}=0, & \forall a \in \mathcal{A} \backslash A_{p}
\end{array}
$$

where $\lambda$ is a step size which will be determined by the following algorithm.

\section{Modified Origin-based Algorithm}

\section{Initialization}

For $p$ from 1 to $N$

$A_{p}=$ tree of minimum cost paths from origin $\mathrm{p}$

$x^{p}=$ all-or-nothing assignment using $A_{p}$

Calculate $\alpha^{p}$ by using (2)

End for

\section{Main loop}

While $x$ does not satisfy the convergence condition do

For $p$ from 1 to $N$

Update restricting subnetwork $A_{p}$

Update origin-based link flows for origin $p$

End for

End while

Update restricting subnetwork for origin $p$ 
Remove unused links from $A_{p}$

Compute maximum cost $u_{i}$ from $p$ to $i$ for all $i \in \mathcal{N}$

For $a=\left[a_{t}, a_{h}\right]$ in $\mathcal{A}$

If $u_{a_{t}}<u_{a_{h}}$ add link a to $A_{p}$

End for

Find topological order for new $A_{p}$

Update data structures

\section{Update origin-based link flows for origin $p$}

Using (14) and (18) to compute average costs and Hessian approximations

For step size $\lambda=2^{-k}, k=0,1,2,3, \cdots$

Using (19) to compute flow shifts $\Delta \alpha^{p}$ for $\lambda$

Projection and aggregate flow shifts

If new value of objective function is less than the old then stop

End for

Apply flow shifts

Update total link flows and link costs.

\section{Numerical Examples}

Below we will illustrate both OBA and MOBA by the following networks whose basic characteristics are presented in Table 1. All programs are coded in $C \sharp$ and executed on a microcomputer with P4 2.0G, 512M. For fair comparison, common data structures for storing network topology and links and same convergence criterion are used in both OBA and the modified algorithm(MOBA). The following steps are used for the comparison of algorithm performance.

Table 1. Basic Characteristics of Test Urban Networks

\begin{tabular}{|c|c|c|c|c|}
\hline Netork & Origin Destination & Node & Link & OD Pair \\
\hline Sioux Falls & 24 & 24 & 76 & $\overline{528}$ \\
\hline Barcelona & 108 & 930 & 2,522 & 7,922 \\
\hline
\end{tabular}

Step 1 . Solve TAP by LCFW (a modified Frank Wlofe algorithm) 8 , with very large number of iteration and highly strict convergence criterion. Tag its final solution as Ideal Optimal Solution(IOS) and denote it with $x^{I}=\left(\cdots, x_{a}^{I}, \cdots\right)$. The intuitive reason for choosing LCFW is that it outperforms the other algorithms(including FW, GP etc. 8]) and it can avoid path enumerations.

Step 2. Considering that there is only one flow pattern that minimizes program (11) [3], we use the following convergence criterion in both OBA and MOBA.

$$
\varepsilon=\frac{\sum_{a \in \mathcal{A}}\left(x_{a}(n)-x_{a}^{I}\right)^{2}}{\sum_{a \in \mathcal{A}} x_{a}^{I^{2}}}
$$

where $x_{a}(n)$ is the link flows of the $n$-th iteration of the algorithms. When $\varepsilon$ is less than a given small positive constant such as $1.0 \times 10^{-n}, n=$ $0,1,2, \cdots$, the algorithms will stop. 


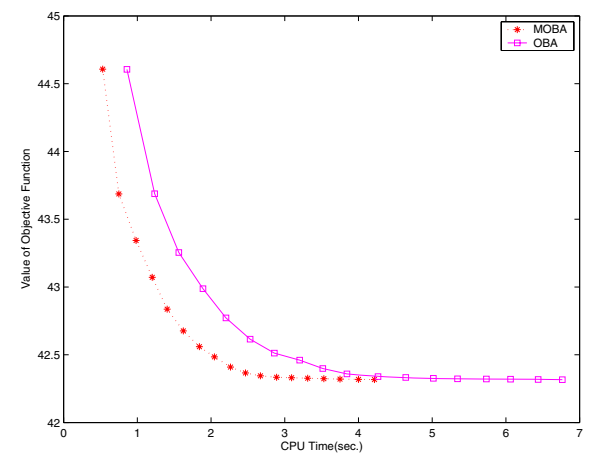

Fig. 1. OFV-CPU Time for Sioux Falls

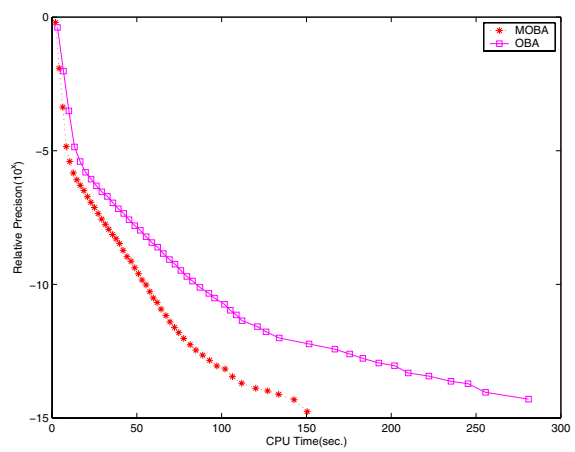

Fig. 3. $\log \varepsilon$-CPU Time for Sioux Falls

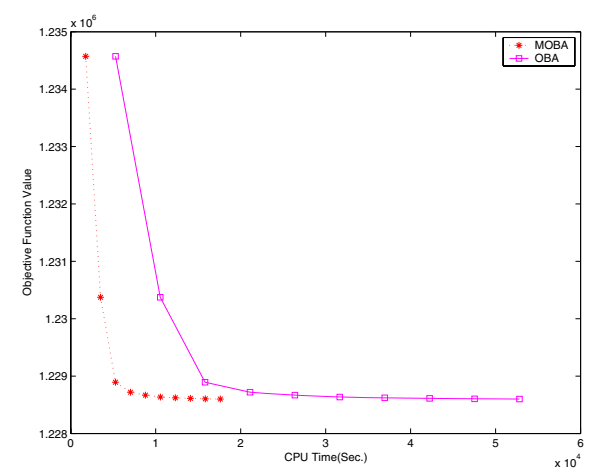

Fig. 2. OFV-CPU Time for Barselona

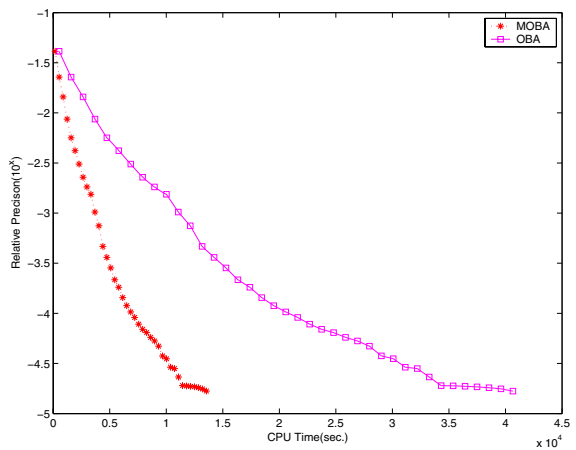

Fig. 4. $\log \varepsilon$-CPU Time for Barselona

Figure 1 and 2 show the relationship between the objective function value(OFV) and CPU time of the two algorithms for Sioux Falls network and Barcelona network. Figure 3 and 4 show the convergence of the two algorithms as measured by $\log \varepsilon$ vs. CPU time for those two real networks. From those four fights, we knew the performance of the modified origin based algorithm is better than that of origin-based algorithm. The modified algorithm is superior to the origin-basd algorithm in convergence and calculation time. At the same precision, the CPU time of the modified algorithm is only one third of the origin-based algorithm, even less than that. And the modified algorithm can solve the traffic assignment problem for large-scale network in an ideal time.

\section{Conclusion}

In this paper we provide a modififed origin-based algorithm based on esitimation of the lower and upper bounds of the Hessian matrix for, which can be calculated without path enumeration. In this algorithm we use the lower bound of Hessian matrix to approximate the direction of the origin-based algorithm. According 
to our computational results, the modified origin-based algorithm(MOBA) improves the convergence performance greatly. The results indicate that MOBA can deliver better and more reliable convergence than OBA and saves much more CPU time especially when large-scale networks are being considered.

\section{References}

1. Wardrop, J.G.: Some theoretical aspects of road traffic research. In: Proceedings of the institute of civil engineers, Part II, pp. 325-378 (1952)

2. Beckmann, M., Mcguire, C.B., Winster, C.B.: Studies in the economics of transportation. Yale University Press, New Heaven (1956)

3. Sheffi, Y.: Urban transportation networks: equilibrium analysis with mathematical programming methods. Prentice Hall, Inc., Englewood Cliffs (1984)

4. Leblanc, L.J., Morlok, E.K., Pierskalla, W.: An efficient approach to solving the road network equilibrium traffic assignment problem. Transportation Research 9, 309-318 (1975)

5. Leblanc, L.J., Helgason, R.V., Boyce, D.E.: Improved efficiency of the FrankWolfe algorithm for convex network problems. Transportation Science 19, 445-462 (1985)

6. Fukushima, M.: A modified frankwolfe algorithm for solving the traffic assignment problem. Transportation Research Part B 18(2), 169-177 (1984)

7. Anders, W., Carmen, O.: Accelerating convergence of the frankwolfe algorithm. Transportation Research Part B 19(2), 113-122 (1985)

8. Lee, D.H., Nie, Y.: Accelerating strategies and computational studies of the FrankWolfe algorithm for the traffic assignment problem. Transportation Research Record 1771, 97-105 (2001)

9. Gao, Z.Y., Lam, W.H.K., Wong, S.C., Yang, H.: The convergence of equilibrium algorithms with non-monotone line search technique. Applied Mathematics and Computation 148, 1-13 (2004)

10. Larsson, T., Patriksson, M.: Simplicial decomposition with disaggregated representation for the traffic assignment problem. Transportation Science 26(1), 4-17 (1992)

11. Bertsekas, D.: On the Goldstein-Levitin-Polyak gradient projection method. IEEE Transaction on Automatic Control 21, 174-183 (1976)

12. Jayakrishnan, R., Tsai, W.K., Prashker, J.N.: Faster path-based algorithm for traffic assignment. Transportation Research Record 1443, 75-83 (1994)

13. Lee, D.H., Nie, Y., Chen, A.: A conjugate gradient projection algorithm for the traffic assignment problem. Mathematical and computer modeling 37, 863-878 (2003)

14. Bar-Gera, H.: Origin-based algorithm for the traffic assignment problem. Transportation Science 36(4), 398-417 (2002) 